\title{
EFFECT OF CARAWAY SEEDS SIEVING (Carum carvi L.) IN FEED REX RABBITS ON: 2. PRODUCTIVE AND CARCASS CHARACTERISTICS
}

\author{
Azza M.M. Badr \\ Regional Center for Food and Feed, Agriculture Research Center, Giza, Egypt. \\ E mail: Azzabadr805@yahoo.com
}

(Received 5/6/2019, accepted 29/7/2019)

\section{SUMMARY}

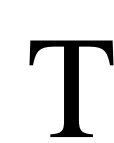

hirty-six of unsexed Rex rabbits breed aged 5-6 weeks and average weight $687.25 \pm 2.75 \mathrm{~g}$ were Trandomly assigned into four groups, (9 rabbits in each) in 3 replicates. The aim of this study to investigate the impact of inclusion caraway seeds sieving (CSS) in rabbit diets on their productive and carcass characteristics. The experimental group rabbits were classified as the following: first group was fed basal diet that contained zero level caraway seeds sieving (CSS) and served as control group $\left(\mathrm{R}_{1}\right)$, second group was fed diet contained $2 \% \mathrm{CSS}$, third group was fed diet contained $4 \%$ CSS and fourth group was fed diet contained $6 \%$ CSS. The results cleared that the experimental diets were isonitrogenous but differed in energy content. Crude protein ranged from $20.35 \%$ to $20.88 \%$, while the values of gross energy (GE) ranged from 4190 to $4308 \mathrm{kcal} / \mathrm{kg} \mathrm{DM}$; meanwhile digestible energy (DE) contents varied from 2090 to $2367 \mathrm{kcal} / \mathrm{kg}$ DM. Animals were slaughtered after 56 days of feeding. Inclusion of CSS significantly increased $(\mathrm{P}<0.05)$ dressing percentages, meanwhile, it had not significantly $(\mathrm{P}>0.05)$ affected on content of digestive tract presented as (full, empty and content). Incorporated CSS in the experimental diets significant increase carcass weight (CW1) by (30.31\%, $50.62 \%$ and $32.66 \%)$; carcass weight (CW2) by $(29.06 \%, 49.41 \%$ and $31.79 \%)$ and carcass weight (CW3) by $\left(25.46 \%, 21.20 \%\right.$ and $6.97 \%$, ) for $\left(\mathrm{R}_{2}, \mathrm{R}_{3}\right.$ and $\left.\mathrm{R}_{4}\right)$, respectively comparing with the control $\left(R_{1}\right)$. The best results of carcass were recorded with rabbits fed $R_{3}$ that contained $4 \%$ CSS. The best result of dressing percentages was observed with rabbits received $\left(\mathrm{R}_{3}\right)$. Total external offal's weight was affected by dietary diets by $16.50 \%, 31.37 \%$ and $25.39 \%$, for groups $\mathrm{R}_{2}, \mathrm{R}_{3}$ and $\mathrm{R}_{4}$, respectively in comparison with the control group. Internal offal's (giblets) weights includes (liver, heart, kidneys, testes, spleen and lungs) and total giblet weight was not affected by incorporated CSS in rabbit diets. Carcass cuts weight includes (fore, middle and hind parts) were improved with rabbits fed $\mathrm{R}_{3}$. Also, physical and chemical analysis of ribs $\left(9,10\right.$ and $\left.11^{\text {th }}\right)$ was significantly affected. From the results obtained it can be mentioned that incorporation caraway seeds sieving (CSS) in rabbit diets up to $4 \%$ could improve their dressing percentages and carcass characteristics without occurred any adverse effect on the other carcass parameters.

Keywords: Caraway seeds sieving, carcass characteristics, dressing percentage, external and internal offal's, digestive tract, physical and chemical characteristics of meat.

\section{INTRODUCTION}

Herbs and herbal extracts contain different phytochemical compounds with biological activity that may provide therapeutic effects. Several herbs help to reduce high blood cholesterol concentration, provide some protection against cancer, and/or stimulate the immune system. Furthermore, it was found that a diet in which culinary herbs are used generously to flavor food provides a variety of active phytochemicals which promote health and protect against chronic diseases (Abdo et al. 2003).

Nowadays, herbal medicine is a growing area as an alternative medicine for human being and many manufactured drugs derived originally from plant compounds have wide range uses. The medicinal plants are rich in a wide variety of nutrients and they may be used as chemotherapeutics and feed additives (Chang, 2000). The use of medicinal plants as a natural feed additive in fish diets is becoming useful rather than classic chemicals, which may have an accumulative effect on human health. They can be used as an attractant to increase feed intake, improve feed utilization, and increase weight gain (Xiang and Zhou 2000). 
Beneficial effects of herbal extracts or active substances in animal nutrition may include the stimulation of appetite and feed intake, the improvement of endogenous digestive enzyme secretion, activation of immune response and antibacterial, antiviral, antioxidant and antihelminthic actions. Isoprene derivatives, flavonoids, glucosinolates and other plant metabolites may affect the physiological and chemical function of the digestive tract (Rahimi et al. 2011) and increase stimulatory influence particularly on bile secretion and pancreatic enzymes activity (Platel et al. 2002)

Caraway (Carum carvi) fruits possess stimulant, expectorant and antispasmodic effects and is used for stomach aches, constipation, and nausea. It increases the secretion of gastric juice and promotes the discharge of bile, which increases the appetite and has digestive stimulatory effects (Peter 2006). Caraway is recommended by Persian traditional scholars to relieve the flatulence. It acts by improvement of digestive systems and deletion of accumulated gas from gastrointestinal tract, humors from stomach, which also relives the abdominal pain. Caraway is an edible plant with longstanding uses as a dietary herbal supplemented (Valizadih et al. 2007). Caraway has great importance in traditional, as well as in modern medicine (Deepak 2013; El-Soud et al. 2014). It is often used in veterinary medicine, as in human medicine, for the stimulation of ap-petite and feed intake, improvement of endogenous digestive enzyme secretion, activation of immune response and anti-bacterial, antiviral, antioxidant and antihelminthic activities (Hassan and Abdel-Raheem 2013). Wichtl (1994) reported that caraway promotes gastric secretion, stimulates appetite, and is used as a remedy for colic, loss of appetite, and digestive disorders. Moreover, the German E commission reports that caraway seeds and oils have antimicrobial and strongly fungicidal activities and can relieve the feeling of bloating or fullness associated with indigestion and stomach complaints.

Caraway seed is used in meat, food and distillery industries due to its pleasant flavor and intense taste. Its antibacterial and fungicidal properties are important in pharmaceutical applications and also in human and veterinary medicine (Sedlakova et al. 2001). El-Dakar (2004) who used among individuals' different levels of CSM $(0,0.5,1$ and $2 \%$ of dried CSM) on growth, survival, and body composition of hybrid tilapia, O. niloticus $\times$ O. auraus fingerlings $(13 \mathrm{~g})$ fed on $0.5 \%$ caraway seed meal $(\mathrm{CSM})$ diet gave significantly higher body weight and weight gain than those fed on other CSM levels.

So, this work was conducted to study the importance of caraway seed sieving in rabbits diet on their feed intake, dressing percentages, carcass cuts, external and internal offal's (giblets), physical composition and chemical analysis of best ribs and weight and length of digestive tract.

\section{MATERIALS AND METHODS}

\section{Animals, management and feed:}

The experiment was carried out at the privet farm in Giza, Egypt. Thirty-six of unsexed Rex rabbits breed aged 5-6 weeks and average weight $687.25 \pm 2.75 \mathrm{~g}$ were randomly assigned into four groups, 9 for each group in 3 replicates. The trail was done throughout the summer season (year 2018). The rabbits were fed the four tested diets that lasted for 56 days. The basal experimental diet was formulated and pelleted to cover the nutrient requirements of rabbits according to NRC (1977) as shown in Table (1). The experimental groups were classified as the following: First group was fed the basal diet and served as control group $\left(\mathrm{R}_{1}\right)$, second group was fed diet contained $2 \%$ of caraway seed sieving (CSS), $\mathrm{R}_{2}$ ), third group was fed diet contained $4 \% \operatorname{CSS}\left(\mathrm{R}_{3}\right)$ and fourth group was fed diet contained $6 \%$ CSS $\left(\mathrm{R}_{4}\right)$.

Each three rabbit groups were housed in galvanized wire cages $(30$ x 35 x $40 \mathrm{~cm})$. Stainless steel nipples for drinking and feeders allowing recording individual feed intake for each rabbit were supplied for each cage (ad libitum). Rabbits of all groups were kept under the same managerial conditions. Samples of tested diets samples and feces were analyzed according to AOAC (2005) methods. Meanwhile, neutral detergent fiber (NDF), acid detergent fiber (ADF) and acid detergent lignin (ADL) were determined according to Van-Soest et al. (1991). In addition, hemicellulose content was calculated as the difference between NDF and ADF, while, cellulose content was calculated as the difference between ADF and ADL. Feed intake (FI) and live body weight (LBW) values were recorded throughout the experimental growing period to calculate the daily feed intake (DFI), average daily gain (ADG), feed conversion ratio (FCR). 
Egyptian J. Nutrition and Feeds (2019)

Table (1): Composition of the experimental diet (\%).

\begin{tabular}{lcccc}
\hline Item & \multicolumn{4}{c}{ Experimental diets } \\
\cline { 2 - 5 } & $\mathrm{R}_{1}$ & $\mathrm{R}_{2}$ & $\mathrm{R}_{3}$ & $\mathrm{R}_{4}$ \\
& (Control, $0 \%$ CSS $)$ & $(2 \%$ CSS $)$ & (4\% CSS) & (6\% CSS) \\
\hline Yellow Corn & 33 & 33 & 33 & 33 \\
Soybean seeds, meal & 26 & 26 & 26 & 26 \\
berseem hay & 29.77 & 27.77 & 25.77 & 23.77 \\
Sunflower oil, refined & 2.05 & 2.05 & 2.05 & 2.05 \\
Corn Gluten Meal & 1 & 1 & 1 & 1 \\
Caraway seed sieving (CSS) & 0 & 2 & 4 & 6 \\
Calcium phosphate, dibasic & 1.5 & 1.5 & 1.5 & 1.5 \\
Sugar Cane Molasses & 3 & 3 & 3 & 3 \\
Coarse Wheat bran & 1.4 & 1.4 & 1.4 & 1.4 \\
Salt & 0.35 & 0.35 & 0.35 & 0.35 \\
Limestone & 1.15 & 1.15 & 1.15 & 1.15 \\
Methionine & 0.33 & 0.33 & 0.33 & 0.33 \\
L-Lysine HCL 98\% & 0.15 & 0.15 & 0.15 & 0.15 \\
Premix (Vit. \& Min. mixture) & 0.3 & 0.3 & 0.3 & 0.3 \\
Total & 100 & 100 & 100 & 100 \\
\hline R: Control diet. $R_{2}:$ contained $2 \%$ from
\end{tabular}

$R_{1}$ : Control diet. $R_{2}$ : contained $2 \%$ from caraway seeds sieving. $R_{3}$ : contained $4 \%$ from caraway seeds sieving. $R_{4}$ : contained $6 \%$ from caraway seeds sieving. * Vit. \& Min. mixture: Each kilogram of Vit. \& Min. mixture contains: 2000.000 IU Vit. A, 150.000 IU Vita. D, 8.33 g Vit. E, $0.33 \mathrm{~g}$ Vit. K, $0.33 \mathrm{~g}$ Vit. B1, $1.0 \mathrm{~g}$ Vit. B2, $0.33 \mathrm{~g}$ Vit. B6, $8.33 \mathrm{~g}$ Vit.B5, $1.7 \mathrm{mg}$ Vit. B12, $3.33 \mathrm{~g}$ Pantothenic acid, $33 \mathrm{mg}$ Biotin, 0.83g Folic acid, $200 \mathrm{~g}$ Choline chloride, $11.7 \mathrm{~g} \mathrm{Zn}$, $12.5 \mathrm{~g} \mathrm{Fe}, 16.6 \mathrm{mg} \mathrm{Se}, 16.6 \mathrm{mg} \mathrm{Co,} 66.7 \mathrm{~g} \mathrm{Mg}$ and $5 \mathrm{~g} \mathrm{M}$.

\section{Slaughter trials:}

At the end of the experimental period after (56 days) three rabbits from each treatment were randomly chosen and fasted for 12 hours before slaughtering according to Blasco et al., ( 1993) to determine the carcass measurements. Edible offal's (giblets) includes (heart, liver, testes, kidneys, spleen and lungs) were removed and individually weighed. Full and empty weights of digestive tract were recorded, and digestive tract contents were calculated by differences between full and empty digestive tract. Weight and length of digestive tract of the experimental groups were recorded. Weights of carcass, giblets and external offal's were calculated as percentages of body weight at slaughtering (SW). Hot carcass was weighed, and half of rabbit carcass was divided into three parts (fore, middle and hind parts) to determine carcass cuts. Weights of carcass cuts were expressed as percentages of carcass weight $(\mathrm{CW})$. Dressing percentages were calculated as either (Carcass weight/ slaughter weight *100) or (Carcass weight/ empty body weight *100). The 9, 10 and $11^{\text {th }}$ ribs were frozen in polyethylene bags for later chemical analysis. The air-dried samples of ribs were analyzed for DM, EE, CP and ash. Physical composition of the $9^{\text {th }}, 10^{\text {th }}$ and $11^{\text {th }}$ ribs was divided into lean, bone and fat weight, g. Weights of external offal's, digestive tract and edible offal's (giblets) were calculated as percentages of slaughtering weight (SW). While, physical composition (lean, bone and fat) were calculated as percentages of best ribs weight (RW). On the other hand, carcass cuts were calculated as percentages of carcass weight $(\mathrm{CW})$.

\section{Statistical analysis:}

Collected data were subjected to statistical analysis of variance using SAS system (2002). Duncan's Multiple Range Test (1955) was used to separate means when the dietary treatment effect was significant.

\section{RESULTS AND DISCUSSION}

\section{Chemical analysis of the experimental diets:}

Chemical analysis and cell wall constituents of the experimental diets are presented in Table (2). Cleared that the experimental diets were isonitrogenous but differed in their energy content. These are related to add caraway seed sieving in the tested diets four tested diets. Crude protein ranged from 
$20.35 \%$ to $21.40 \%$, while EE content was ranged from $4.71 \%$ to $5.78 \%$ among the four experimental diets. In addition, $\mathrm{CF}$ content ranged from 8.28 to $10.42 \%$; meanwhile NFE content ranged from 52.32 to $53.92 \%$ for the same four tested diets. The values of gross energy (GE) ranged from 4190 to $4308 \mathrm{kcal} /$ $\mathrm{kg} \mathrm{DM}$; meanwhile digestible energy (DE) varied from 2090 to $2367 \mathrm{kcal} / \mathrm{kg}$ DM. Non-fibrous carbohydrates (NFC) ranged from 45.49 to $49.17 \%$ among the four experimental diets. Digestible energy (Kcal/kg DM) content of experimental diets $\left(R_{2}\right.$ to $\left.R_{3}\right)$ were increased compared to $\left(R_{1}\right.$ and $\left.R_{4}\right)$. The corresponding values of digestible energy were (2329 and $2367 \mathrm{Kcal} / \mathrm{kg} \mathrm{DM})$ for $\mathrm{R}_{2}$ and $\mathrm{R}_{3}$ vs. (2267 and $2090 \mathrm{Kcal} / \mathrm{kg} \mathrm{DM}$ ) for $\mathrm{R}_{1}$ and $\mathrm{R}_{4}$, respectively. Meanwhile, the highest value of non-fibrous carbohydrates (NFC) was recorded by $\mathrm{R}_{3}$ (49.17). Cell wall constituents (NDF, ADF, ADL, hemicellulose and cellulose) contents of the experimental diet were decreased when CSS incorporated in rabbit diets in comparison with the control diet.

Table (2): Chemical composition and cell wall constituents of the experimental diets (\% on dry matter basis).

\begin{tabular}{|c|c|c|c|c|}
\hline \multirow[t]{2}{*}{ Item } & \multicolumn{4}{|c|}{ Experimental diets } \\
\hline & $\begin{array}{c}\mathrm{R}_{1} \\
\text { (Control, 0\% CSS) }\end{array}$ & $\begin{array}{c}\mathrm{R}_{2} \\
(2 \% \mathrm{CSS})\end{array}$ & $\begin{array}{c}\mathrm{R}_{3} \\
(4 \% \mathrm{CSS})\end{array}$ & $\begin{array}{c}\mathrm{R}_{4} \\
(6 \% \mathrm{CSS})\end{array}$ \\
\hline Dry matter (DM) & 93.38 & 91.59 & 93.85 & 93.86 \\
\hline \multicolumn{5}{|l|}{ Chemical analysis \% on DM basis: } \\
\hline Organic matter $(\mathrm{OM})$ & 88.60 & 88.77 & 88.91 & 86.89 \\
\hline Crude protein $(\mathrm{CP})$ & 20.35 & 21.4 & 20.88 & 20.88 \\
\hline Crude fiber $(\mathrm{CF})$ & 10.42 & 9.27 & 8.73 & 8.28 \\
\hline Ether extract (EE) & 4.71 & 5.78 & 5.38 & 5.15 \\
\hline Nitrogen-free extract (NFE) & 53.12 & 52.32 & 53.92 & 52.58 \\
\hline Ash & 11.4 & 11.23 & 11.09 & 13.11 \\
\hline Gross energy (Kcal/kg DM $)^{1}$ & 4229 & 4308 & 4285 & 4190 \\
\hline Digestible energy $(\mathrm{Kcal} / \mathrm{kg} \mathrm{DM})^{2}$ & 2267 & 2329 & 2367 & 2090 \\
\hline Non fibrous carbohydrates (NFC) ${ }^{3}$ & 46.12 & 45.49 & 49.17 & 46.02 \\
\hline \multicolumn{5}{|l|}{ 2- Cell wall constituents (\%): } \\
\hline Neutral detergent fiber (NDF) & 17.42 & 16.1 & 13.48 & 14.84 \\
\hline Acid detergent fiber (ADF) & 12.55 & 11.77 & 10.78 & 10.29 \\
\hline Acid detergent lignin (ADL) & 3.44 & 3.2 & 2.96 & 3 \\
\hline Hemicellulose & 4.87 & 4.32 & 2.69 & 4.55 \\
\hline Cellulose & 9.11 & 8.57 & 7.82 & 7.82 \\
\hline
\end{tabular}

$R_{1}$ : Control diet. $R_{2}$ : contained $2 \%$ from caraway seeds sieving. $R_{3}$ : contained $4 \%$ from caraway seeds sieving. $R_{4}$ : contained $6 \%$ from caraway seeds sieving. ${ }^{1}$ Gross energy (Kcal/kg DM) was calculated according to Blaxter (1968), where, each $g$ of crude protein $(C P)=5.65 \mathrm{kcal}$, each $\mathrm{g}$ of ether extract $(E E)=9.40 \mathrm{kcal}$, and each $\mathrm{g}$ crude fiber $(C F)$ and nitrogen-free extract $(N F E)=4.15 \mathrm{kcal} .{ }^{2}$ Digestible energy $($ Kcal $/ \mathrm{kg} D M)$ was calculated according to Fekete and Gippert (1986) using the following equation: $D E(\mathrm{kcal} / \mathrm{kg} \mathrm{DM})=4253-32.6(C F \%)-144.4$ (total ash).

${ }^{3}$ Non fibrous carbohydrates (NFC) were calculated according to Calsamiglia et al. (1995) using the following equation: $N F C=100-\{C P+E E+A s h+N D F\}$.

\section{Carcass characteristics:}

Effect of the experimental diets on dressing percentages are illustrated in Table (3). Dietary treatment containing caraway seed sieving (CSS) had not significantly affect $(\mathrm{P}>0.05)$ on full, empty and content of digestive tract. However, it significantly increased empty body weight (EBW) comparing to the control. Dietary treatments significant $(\mathrm{P}<0.05)$ increased carcass weight $(\mathrm{CW} 1)$ by $30.31 \%, 50.62 \%$ and $32.66 \%$ for (CW1) and (CW2) by $29.06 \%, 49.41 \%$ and $31.79 \%$, for 2,4 and $6 \%$ CSS, respectively. Carcass weight (CW3) that includes edible offal's (Liver, heart, kidneys, spleen, testes and lungs) were improved by $25.46 \%, 21.20 \%$ and $6.97 \%$ for $\left(R_{2}, R_{3}\right.$ and $\left.R_{4}\right)$, respectively in comparison with the control $\left(R_{1}\right)$. The best results of carcass were obtained by feeding rabbits on $\mathrm{R}_{3}$ that contained $4 \%$ CSS. Dietary treatments had significant effect $(\mathrm{P}<0.05)$ on dressing percentages $(\mathrm{DP}) \%$. In addition to, the best result recorded with group rabbits that fed diet $\mathrm{R}_{3}$. These results agreement with obtained by Ibrahim et al. (2000) noted that adding $0.5 \%$ of sweet basil or oregano in rabbit diets significantly $(\mathrm{P}<0.05)$ increased their dressing percentages and giblets. But Jafari (2011) showed that the not effect of dietary supplementation when applying different levels of caraway seed powder $(1.0 \%, 1.5 \%$ and $2.0 \%)$, on performance and carcass traits. 
Egyptian J. Nutrition and Feeds (2019)

Table (3): Dressing percentages of the experimental rabbits groups fed different diets.

\begin{tabular}{|c|c|c|c|c|}
\hline \multirow[t]{2}{*}{ Item } & \multicolumn{4}{|c|}{ Experimental diets } \\
\hline & $\begin{array}{c}\mathrm{R}_{1} \text { (Control, } \\
0 \% \text { CSS })\end{array}$ & $\begin{array}{c}\mathrm{R}_{2} \\
(2 \% \mathrm{CSS})\end{array}$ & $\begin{array}{c}\mathrm{R}_{3} \\
(4 \% \mathrm{CSS})\end{array}$ & $\begin{array}{c}\mathrm{R}_{4} \\
(6 \% \mathrm{CSS})\end{array}$ \\
\hline $\begin{array}{l}\text { Slaughter weight }(\mathrm{SW}), \mathrm{g} \text { : } \\
\text { Digestive tract }\end{array}$ & $1785.67^{\mathrm{b}} \pm 34.37$ & $2181^{\mathrm{a}} \pm 133.01$ & $2446.33^{\mathrm{a}} \pm 128.97$ & $2272.67^{\mathrm{a}} \pm 59.68$ \\
\hline Full weight, g & $285.34 \pm 4.98$ & $286.91 \pm 12.01$ & $288.33 \pm 44.19$ & $322.67 \pm 43.48$ \\
\hline Empty weight, g & $109.34 \pm 5.93$ & $107.33 \pm 7.84$ & $114.33 \pm 7.88$ & $128.67 \pm 5.93$ \\
\hline Content weight, $\mathrm{g}$ & $176 \pm 7.32$ & $173.33 \pm 10.48$ & $171.67 \pm 36.86$ & $194.67 \pm 33.32$ \\
\hline Empty body weight (EBW), g & $1500.33^{\mathrm{b}} \pm 30.34$ & $1895.09^{\mathrm{a}} \pm 121.30$ & $2158^{\mathrm{a}} \pm 88.06$ & $1949.5^{\mathrm{a}} \pm 98.86$ \\
\hline Head eight, $g$ & $106.67^{c} \pm 1.20$ & $126.33^{\mathrm{b}} \pm 6.12$ & $148.67^{\mathrm{a}} \pm 3.18$ & $132.67^{\mathrm{b}} \pm 6.06$ \\
\hline Carcass weight (CW1) & $907.33^{\mathrm{b}} \pm 33.79$ & $1182.33^{\mathrm{a}} \pm 96.80$ & $1366.66^{\mathrm{a}} \pm 57.67$ & $1203.67^{\mathrm{a}} \pm 46.62$ \\
\hline Carcass weight (CW2) & $1014^{\mathrm{b}} \pm 33.83$ & $1308.67^{\mathrm{a}} \pm 102.52$ & $1515^{\mathrm{a}} \pm 55.01$ & $1336.33^{\mathrm{a}} \pm 50.44$ \\
\hline Carcass weight (CW3) & $1115.67^{\mathrm{b}} \pm 36.86$ & $1399.67^{\mathrm{a}} \pm 92.15$ & $1352.16^{\mathrm{a}} \pm 61.20$ & $1193.45^{\mathrm{ab}} \pm 49.04$ \\
\hline \multicolumn{5}{|l|}{ Dressing percentages $(D P) \%$ : } \\
\hline DP1 & $50.79^{\mathrm{b}} \pm 1.06$ & $54.08^{\mathrm{ab}} \pm 1.11$ & $55.933^{\mathrm{a}} \pm 1.05$ & $58.78^{\mathrm{ab}} \pm 0.70$ \\
\hline DP2 & $56.76^{\mathrm{b}} \pm 1.02$ & $59.88^{\mathrm{ab}} \pm 1.05$ & $62.06^{\mathrm{a}} \pm 1.45$ & $52.48^{\mathrm{b}} \pm 0.88$ \\
\hline DP3 & $62.46^{\mathrm{a}} \pm 1.20$ & $64.14^{\mathrm{a}} \pm 0.71$ & $55.36^{\mathrm{a}} \pm 1.08$ & $61.82 \pm 0.76$ \\
\hline DP4 & $60.44 \pm 1.15$ & $62.253 \pm 1.09$ & $63.31 \pm 0.40$ & $68.65 \pm 70.64$ \\
\hline DP5 & $67.56 \pm 1.08$ & $68.937 \pm 0.99$ & $70.233 \pm 0.61$ & $61.28^{\mathrm{b}} \pm 0.59$ \\
\hline DP6 & $74.33^{\mathrm{a}} \pm 1.28$ & $73.84^{\mathrm{a}} \pm 0.64$ & $62.67^{\mathrm{b}} \pm 0.37$ & $58.78^{\mathrm{ab}} \pm 0.70$ \\
\hline \multicolumn{5}{|c|}{$\begin{array}{l}\text { a and b: Mean in the same row having different superscripts differ significantly }(P<0.05) . R_{1}: \text { Control diet. } R_{2}: \\
\text { contained } 2 \% \text { from caraway seeds sieving. } R_{3}: \text { contained } 4 \% \text { from caraway seeds sieving. } R_{4}: \text { contained } 6 \% \text { from } \\
\text { caraway seeds sieving. EBW: Empty body weight }=\text { Slaughter weight - digestive tract content. CW1: Carcass weight. } \\
C W 2: \text { Carcass weight }+ \text { head. CW3: Carcass weight }+ \text { head }+ \text { edible offal's include (Liver, heart, kidneys, testes, } \\
\text { lungs and spleen }) . D P 1: \text { Dressing percentages calculated as }(C W 1 / S W * 100) . \quad D P 2: \text { Dressing percentages } \\
\text { calculated as }(C W 2 / S W * 100) \text {. DP3: Dressing percentages calculated as }(C W 3 / S W * 100) . \text { DP4: Dressing } \\
\text { percentages calculated as }(C W 1 / E B W * 100) . D P 5: \text { Dressing percentages calculated as }(C W 2 / E B W * 100) \text {. DP6: } \\
\text { Dressing percentages calculated as }(C W 3+E B W * 100) \text {. }\end{array}$} \\
\hline
\end{tabular}

\section{External and internal offal's (giblets):}

External and internal offal's (giblets) that presented in Table (4) indicated that dietary treatments had significant $(\mathrm{P}<0.05)$ increased effect on external offal's included ( head, fur, legs, ears, and blood). While, the same parameters expressed as \% of SW were not affected by incorporated CSS. The highest values were recorded with the $\mathrm{R}_{3}$ that contained $4 \% \mathrm{CSS}$. Total weight of external offal's had significant positive effect which treatments containing caraway seed sieving by $16.50 \%, 31.37 \%$ and $25.39 \%$, for diets $\mathrm{R}_{2}$, $\mathrm{R}_{3}$ and $\mathrm{R}_{4}$; respectively in comparison with the control $\left(\mathrm{R}_{1}\right)$.

On the other hand, data of Table (4) showed that except for heart eight, the other internal offal's (giblets) that included (liver, kidneys, testes, spleen and lungs) and total giblet weight were not significant affected by inclusion CSS in rabbit diets. Meanwhile, except for liver and testes the other internal offal's (giblets) that includes (heart, kidneys and lungs) and total giblets that expressed as \% of SW except were significant affected by incorporated CSS in rabbit diets. These results agreement with those obtained by Omer et al. (2013) who noted that fennel (Foeniculum vulgare) or oregano (Origanum vulgare L) had no significant effect $(\mathrm{P}>0.05)$ on slaughter weight $(\mathrm{SW})$; full, empty. and content of digestive tract presented as (weight, or \% of SW) of rabbits. Also, Radwan and Abdel-Khalek (2007) indicated that relative to the slaughter weight, hot carcass, giblets, and total edible parts percentage, were not significantly affected by supplement $0.5 \%$ or $1 \%$ herb mixture composed of equal parts of sage+ oregano+sweet basal. In addition, Çabuk et al. (2006) showed that carcass yield and some internal organ weights such as the liver, pancreas, proventriculus, gizzard and small intestine were not affected by the addition of the essential oil mixture to the diet.

Omer et al. (2013) showed that fennel or oregano had no significant effect on external offal's included (head, fur, legs, ears, and blood) that presented as $\%$ of SW and except for spleen that was significant $(\mathrm{P}<0.05)$ affected by treatments. Also, there were no significant effect by on the other parameters of internal offal's (giblets) that includes (liver, heart, kidneys, testes and lungs). 
Table (4): Effect of experimental diets on external and internal offal's (Giblets) of rabbits.

\begin{tabular}{|c|c|c|c|c|c|}
\hline \multirow{2}{*}{\multicolumn{2}{|c|}{ Item }} & \multicolumn{4}{|c|}{ Experimental diets } \\
\hline & & $\begin{array}{c}\mathrm{R}_{1} \text { (Control, } \\
0 \% \text { CSS) }\end{array}$ & $\begin{array}{c}\mathrm{R}_{2} \\
(2 \% \mathrm{CSS})\end{array}$ & $\begin{array}{c}\mathrm{R}_{3} \\
(4 \% \mathrm{CSS})\end{array}$ & $\begin{array}{c}\mathrm{R}_{4} \\
(6 \% \mathrm{CSS})\end{array}$ \\
\hline \multicolumn{2}{|c|}{ Slaughter weight (SW), g } & $\begin{array}{c}1785.67^{\mathrm{b}} \pm \\
34.37\end{array}$ & $2181^{\mathrm{a}} \pm 133.01$ & $2446.33^{\mathrm{a}} \pm 128.97$ & $2272.67^{\mathrm{a}} \pm 59.68$ \\
\hline \multicolumn{6}{|l|}{ External offal's } \\
\hline \multirow{2}{*}{ Head } & weight, $g$ & $106.67^{c} \pm 1.20$ & $126.33^{\mathrm{b}} \pm 6.12$ & $149^{\mathrm{a}} \pm 3.06$ & $133^{\mathrm{b}} \pm 5.77$ \\
\hline & $\%$ of SW & $5.98 \pm 0.15$ & $5.8 \pm 0.14$ & $6.14 \pm 0.46$ & $5.85 \pm 0.17$ \\
\hline \multirow{4}{*}{$\begin{array}{l}\text { Fur, legs, ears } \\
\text { and blood } \\
\text { Total External } \\
\text { offal's }\end{array}$} & weight, $g$ & $384.33^{\mathrm{b}} \pm 12.57$ & $445.67^{\mathrm{ab}} \pm 29.98$ & $496^{\mathrm{a}} \pm 24.01$ & $482.67^{\mathrm{a}} \pm 39.63$ \\
\hline & $\%$ of SW & $21.52 \pm 0.47$ & $20.42 \pm 0.23$ & $20.30 \pm 0.52$ & $21.18 \pm 1.16$ \\
\hline & weight, $g$ & $491^{\mathrm{b}} \pm 11.53$ & $572^{\mathrm{ab}} \pm 35.92$ & $645^{\mathrm{a}} \pm 21.66$ & $615.67^{\mathrm{a}} \pm 44.28$ \\
\hline & $\%$ of SW & $28.30 \pm 0.41$ & $26.85 \pm 0.37$ & $27.08 \pm 0.87$ & $27.68 \pm 1.27$ \\
\hline \multicolumn{6}{|c|}{ Internal offal's (Giblets } \\
\hline \multirow[t]{2}{*}{ Liver } & weight, $\mathrm{g}$ & $47.67 \pm 1.33$ & $50.33 \pm 5.78$ & $53.67 \pm 4.84$ & $47.67 \pm 1.86$ \\
\hline & $\%$ of SW & $2.67^{\mathrm{a}} \pm 0.07$ & $2.29^{\mathrm{b}} \pm 0.14$ & $2.19^{\mathrm{b}} \pm 0.11$ & $2.1 \mathrm{~b} \pm 0.07$ \\
\hline \multirow[t]{2}{*}{ Heart } & weight, $\mathrm{g}$ & $5.67^{\mathrm{b}} \pm 0.33$ & $7^{\mathrm{ab}} \pm 0.58$ & $10.33^{\mathrm{a}} \pm 2.40$ & $8 a^{b} \pm 0.58$ \\
\hline & $\%$ of SW & $0.31 \pm 0.02$ & $0.32 \pm 0.03$ & $0.41 \pm 0.08$ & $0.35 \pm 0.03$ \\
\hline \multirow[t]{2}{*}{ Kidneys } & weight, $g$ & $24.67 \pm 2.33$ & $25.33 \pm 1.67$ & $37.33 \pm 5.49$ & $33.33 \pm 6.96$ \\
\hline & $\%$ of SW & $1.38 \pm 0.12$ & 4.37 & $1.52 \pm 0.19$ & $1.47 \pm 0.31$ \\
\hline \multirow[t]{2}{*}{ Spleen } & weight, $\mathrm{g}$ & $3 \pm 0.0$ & $3.67 \pm 0.33$ & $4 \pm 1.0$ & $2.67 \pm 0.33$ \\
\hline & $\%$ of SW & $0.17 \pm 0.0$ & $0.17 \pm 0.03$ & $0.16 \pm 0.04$ & $0.12 \pm 0.02$ \\
\hline \multirow[t]{2}{*}{ Testes } & weight, $\mathrm{g}$ & $9 \pm 0.58$ & $9.67 \pm 0.33$ & $10 \pm 0.58$ & $9.33 \pm 0.33$ \\
\hline & $\%$ of SW & $0.5 .06^{\mathrm{a}} \pm 0.03$ & $0.45^{\mathrm{ab}} \pm 0.02$ & $0.41^{\mathrm{b}} \pm 0.03$ & $0.41^{\mathrm{b}} \pm 0.01$ \\
\hline \multirow[t]{2}{*}{ Lungs } & weight, $g$ & $11.67 \pm 0.33$ & $14.33 \pm 0.88$ & $14.33 \pm 0.33$ & $13 \pm 1.53$ \\
\hline & $\%$ of SW & $0.65 \pm 0.03$ & $0.66 \pm 0.02$ & $0.59 \pm 0.03$ & $0.57 \pm 0.05$ \\
\hline \multirow[t]{2}{*}{ Total giblets } & weight, $\mathrm{g}$ & $101.68 \pm 4.37$ & $110.33 \pm 8.19$ & $129.66 \pm 12.39$ & $114 \pm 7.49$ \\
\hline & $\%$ of SW & $5.69 \pm 0.24$ & $5.06 \pm 0.17$ & $5.28 \pm 0.29$ & $5.02 \pm 0.33$ \\
\hline
\end{tabular}

$a, b$ and $c$ : Means in the same row having different superscripts differ significantly $(P<0.05) . R_{1}$ : Control diet. $R_{2}$ : contained $2 \%$ from caraway seeds sieving. $R_{3}$ : contained $4 \%$ from caraway seeds sieving. $R_{4}$ : contained $6 \%$ from caraway seeds sieving.

\section{Digestive tract (weight and length):}

Data of Tables (5 and 6) showed that dietary treatments had no significant effect $(\mathrm{P}>0.05)$ on length of digestive tract includes (stomach, small \& large intestine and caecum) and digestive tract weights that divided into (full, empty and content).

Stomach empty weight recorded the higher values $27.33 \%$ and $24.67 \%$ of SW for rabbits fed $\mathrm{R}_{3}$ and $\mathrm{R}_{4}$ diets, followed by $\mathrm{R}_{2}$ that recorded $23 \%$ of $\mathrm{SW}$ in comparison with control $\left(\mathrm{R}_{1}\right)$ that recorded $17 \%$ of SW.

Full and empty small intestine and empty \% of slaughter weight on small intestine had significant effect between diets, the best result was recorded with $\mathrm{R}_{4}$, the values were $103.67 \mathrm{gm}$ and $64 \mathrm{gm}$, respectively.However, full, empty and content $\%$ of slaughter weight for cecum were decreased comparing with the control $\left(\mathrm{R}_{1}\right)$. Dietary treatments had no significant effect $(\mathrm{P}>0.05)$ on full, empty and content of digestive tract weight and that expressed as \% of SW. These results agree with those found by Omer et al. (2010) who observed that adding $0.5 \%$ lemongrass or active dried yeast in rabbit diets had no significant effect $(\mathrm{P}>0.05)$ on full, empty and content of digestive tract weight. Also, Abd-El-Hady (2014) reported that feeding weaned rabbits diets contained 0,300 and 400 gm digestarom \{ (contained active components: menthol (3.00\% of peppermint), anethol ( $0.45 \%$ of anise, fennel) and carvon $(0.035 \%$ of caraway)/ ton $\}$ did not significantly affect full stomach, full intestine weight and percentages as $\%$ of slaughter weight and intestine length. Dietary treatments except for stomach length had no significant effect on total digestive tract, caecum, small and large intestinal length (Table 6). 
Table (5): Digestive tract weights of the experimental rabbits groups.

\begin{tabular}{|c|c|c|c|c|c|c|}
\hline \multirow{2}{*}{\multicolumn{3}{|c|}{ Item }} & \multirow{2}{*}{\multicolumn{4}{|c|}{ Experimental diets (\%) }} \\
\hline & & & & & & \\
\hline Slaugh & rweight & W) $\sigma$ & $\begin{array}{c}\mathrm{R}_{1} \text { (Control, } \\
0 \% \text { CSS }) \\
1785.67^{\mathrm{b}} \pm 34.37\end{array}$ & $\begin{array}{c}\mathrm{R}_{2} \\
(2 \% \mathrm{CSS}) \\
2181^{\mathrm{a}} \pm 133.01\end{array}$ & $\begin{array}{c}\mathrm{R}_{3} \\
(4 \% \mathrm{CSS}) \\
2446.33^{\mathrm{a}} \pm 128.97\end{array}$ & $\begin{array}{c}\mathrm{R}_{4} \\
(6 \% \mathrm{CSS}) \\
2272.67^{\mathrm{a}} \pm 59.68\end{array}$ \\
\hline \multirow{5}{*}{ 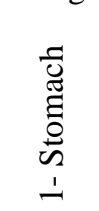 } & \multirow{2}{*}{ Full } & Weight, $g$ & $90.67 \pm 2.85$ & $68.67 \pm 4.33$ & $70 \pm 14.15$ & $79.33 \pm 15.15$ \\
\hline & & $\%$ of SW & $5.08 \pm 0.12$ & $3.16 \pm 0.25$ & $2.82 \pm 0.44$ & $3.52 \pm 0.76$ \\
\hline & \multirow{2}{*}{ Empty } & Weight, $g$ & $17^{\mathrm{b}} \pm 0.0$ & $23^{\mathrm{ab}} \pm 3.21$ & $27.33^{\mathrm{a}} \pm 1.33$ & $24.67^{\mathrm{a}} \pm 2.33$ \\
\hline & & $\%$ of SW & $0.95 \pm 0.02$ & $1.05 \pm 0.10$ & $1.12 \pm 0.07$ & $1.09 \pm 0.12$ \\
\hline & \multirow[t]{2}{*}{ Content } & Weight, $g$ & $73.67 \pm 2.85$ & $45.67 \pm 5.78$ & $42.67 \pm 14.17$ & $54.67 \pm 13.54$ \\
\hline \multirow{7}{*}{ 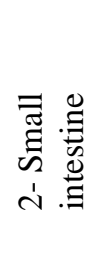 } & & $\%$ of SW & $4.12 \pm 0.12$ & $2.12 \pm 0.35$ & $1.69 \pm 0.49$ & $2.43 \pm 0.65$ \\
\hline & \multirow{2}{*}{ Full } & Weight, g & $65^{\mathrm{b}} \pm 0.58$ & $85.67^{\mathrm{ab}} \pm 0.17$ & $81.67^{\mathrm{ab}} \pm 10.17$ & $103.67^{\mathrm{a}} \pm 6.98$ \\
\hline & & $\%$ of SW & $3.64 \pm 0.04$ & $3.94 \pm 0.24$ & $3.32 \pm 0.32$ & $4.58 \pm 0.42$ \\
\hline & \multirow{2}{*}{ Empty } & Weight, g & $45^{\mathrm{b}} \pm 2.89$ & $44.67^{\mathrm{b}} \pm 7.84$ & $48^{\mathrm{b}} \pm 4.51$ & $64^{\mathrm{a}} \pm 1.53$ \\
\hline & & $\%$ of SW & $2.52^{\mathrm{ab}} \pm 0.12$ & $2.05 \mathrm{ab} \pm 0.37$ & $1.96 b \pm 0.23$ & $2.82 \mathrm{a} \pm 0.12$ \\
\hline & \multirow[t]{2}{*}{ Content } & Weight, g & $20 \pm 2.89$ & $41 \pm 3.51$ & $33.67 \pm 13.92$ & $39.67 \pm 6.33$ \\
\hline & & $\%$ of SW & $1.12 \pm 0.16$ & $1.89 \pm 0.17$ & $1.35 \pm 0.55$ & $1.76 \pm 0.32$ \\
\hline \multirow{6}{*}{ 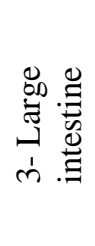 } & \multirow{2}{*}{ Full } & Weight, g & $116.67 \pm 1.76$ & $121 \pm 9.0$ & $126.33 \pm 20.87$ & $128.67 \pm 20.95$ \\
\hline & & $\%$ of SW & $6.53 \pm 0.04$ & $5.54 \pm 0.12$ & $5.11 \pm 0.66$ & $5.71 \pm 1.05$ \\
\hline & \multirow{2}{*}{ Empty } & Weight, g & $39.67 \pm 2.85$ & $32.33 \pm 3.93$ & $32 \pm 2.52$ & $34.33 \pm 7.54$ \\
\hline & & $\%$ of SW & $2.22 \pm 0.15$ & $1.51 \pm 0.26$ & $1.31 \pm 0.05$ & $1.52 \pm 0.36$ \\
\hline & \multirow[t]{2}{*}{ Content } & Weight, g & $77 \pm 2.89$ & $88.67 \pm 12.02$ & $94.33 \pm 18.35$ & $94.33 \pm 14.31$ \\
\hline & & $\%$ of SW & $4.31 \pm 0.17$ & $4.03 \pm 0.29$ & $3.80 \pm 0.61$ & $4.18 \pm 0.73$ \\
\hline \multirow{4}{*}{ ఏ్̃ } & \multirow{2}{*}{ Full } & Weight, $g$ & $13 \pm 0.0$ & $10.67 \pm 0.67$ & $10.33 \pm 2.40$ & $11 \pm 1.15$ \\
\hline & & $\%$ of SW & $0.73^{\mathrm{a}} \pm 0.01$ & $0.49^{\mathrm{b}} \pm 0.06$ & $0.42^{\mathrm{b}} \pm 0.08$ & $0.49^{\mathrm{b}} \pm 0.06$ \\
\hline & \multirow{2}{*}{ Empty } & Weight, g & $7.67^{\mathrm{a}} \pm 0.33$ & $7.33^{\mathrm{a}} \pm 0.67$ & $7.33 \mathrm{a} \pm 0.88$ & $5^{\mathrm{b}} \pm 0.58$ \\
\hline & & $\%$ of SW & $0.43^{\mathrm{a}} \pm 0.02$ & $0.34^{\mathrm{ab}} \pm 0.05$ & $0.30^{\mathrm{bc}} \pm 0.02$ & $0.22^{c} \pm 0.03$ \\
\hline \multirow[t]{2}{*}{$\dot{t}$} & \multirow[t]{2}{*}{ Content } & Weight, $g$ & $5.33 \pm 0.33$ & $3.33 \pm 0.67$ & $3.0 \pm 1.53$ & $6 \pm 0.58$ \\
\hline & & $\%$ of SW & $0.30^{\mathrm{a}} \pm 0.02$ & $0.15^{b c} \pm 0.03$ & $0.12^{c} \pm 1.53$ & $0.27^{\mathrm{ab}} \pm 0.03$ \\
\hline \multirow{6}{*}{ 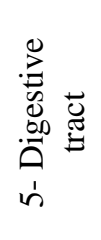 } & \multirow{2}{*}{ Full } & Weight, $g$ & $285.33 \pm 4.98$ & $286 \pm 12.01$ & $288.33 \pm 44.19$ & $323.33 \pm 43.48$ \\
\hline & & $\%$ of SW & $15.98 \pm 0.17$ & $13.15 \pm 0.29$ & $11.66 \pm 1.31$ & $14.33 \pm 2.24$ \\
\hline & \multirow{2}{*}{ Empty } & Weight, g & $109.34 \pm 5.93$ & $112.67 \pm 7.84$ & $116.67 \pm 7.88$ & $128.67 \pm 5.93$ \\
\hline & & $\%$ of SW & $6.12 \pm 0.27$ & $5.17 \pm 0.28$ & $4.76 \pm 0.07$ & $5.68 \pm 0.62$ \\
\hline & \multirow[t]{2}{*}{ Content } & Weight, g & $176 \pm 7.32$ & $173.33 \pm 10.48$ & $171.67 \pm 36.86$ & $194.67 \pm 33.32$ \\
\hline & & $\%$ of SW & $9.86 \pm 0.44$ & $7.97 \pm 0.45$ & $6.90 \pm 1.24$ & $8.65 \pm 1.67$ \\
\hline
\end{tabular}

Table (6): Length of digestive tract of the experimental rabbits groups.

\begin{tabular}{lcccc}
\hline Item & \multicolumn{4}{c}{ Experimental diets } \\
\cline { 2 - 5 } & $\mathrm{R}_{1}$ (Control, & $\mathrm{R}_{2}$ & $\mathrm{R}_{3}$ & $\mathrm{R}_{4}$ \\
& $0 \%$ CSS $)$ & $(2 \%$ CSS $)$ & $(4 \%$ CSS $)$ & $(6 \%$ CSS $)$ \\
\hline Digestive tract length, cm & $339.43 \pm 56.32$ & $408.77 \pm 2.80$ & $382.4 \pm 26.92$ & $402.83 \pm 25.67$ \\
Stomach, cm & $23.5^{\mathrm{a}} \pm 1.04$ & $17.33^{\mathrm{b}} \pm 1.53$ & $15.5^{\mathrm{b} \pm} 1.61$ & $16^{\mathrm{b}} \pm 0.0$ \\
Small intestine length, cm & $244 \pm 57$ & $311.83 \pm 1.74$ & $293 \pm 23.69$ & $313 \pm 21.96$ \\
Large intestine length, cm & $61.33 \pm 1.86$ & $68.67 \pm 1.33$ & $63 \pm 3.00$ & $63.67 \pm 5.17$ \\
caecum, cm & $10.6 \pm 0.21$ & $10.93 \pm 0.35$ & $10.9 \pm 0.97$ & $11.5 \pm 0.58$ \\
\hline
\end{tabular}

$a$ and $b$ : Means in the same row having different superscripts differ significantly $(P<0.05) . R_{1}$ : Control diet. $R_{2}$ : contained $2 \%$ from caraway seeds sieving. $R_{3}$ : contained $4 \%$ from caraway seeds sieving. $R_{4}$ : contained $6 \%$ from caraway seeds sieving.

\section{Carcass cuts and chemical analysis:}

Data illustrated in Table (7) showed that dietary treatment significantly effect $(\mathrm{P}<0.05)$ carcass cuts, that divided into three parts. Weight of fore part was significantly $(\mathrm{P}<0.05)$ increased by incorporated CSS in rabbit diets by $37.09 \%, 57.19$ and $37.06 \%$ for $\left(R_{2}, R_{3}\right.$ and $\left.R_{4}\right)$, respectively compared with the control $\mathrm{R}_{1}$. The best results were recorded with rabbits fed diet $\mathrm{R}_{3}$. In addition, the highest significant weight (expressed as \% of $\mathrm{CW}$ ) was recorded with diet $\mathrm{R}_{3}$ flowed by $\mathrm{R} 2$ and 
$\mathrm{R} 4$ in comparison with the control $\left(\mathrm{R}_{1}\right)$ diet. This increasing reach from $19.23 \%$ in control diet to $21.46,22.15 \%$ and $20.71 \%$ for $\mathrm{R}_{2}, \mathrm{R}_{3}$ and $\mathrm{R}_{4}$, respectively. Also, dietary treatment increased the middle part by $26.08,42.43 \%$ and $32.35 \%$ for $\mathrm{R}_{2}, \mathrm{R}_{3}$ and $\mathrm{R}_{4}$, respectively. Meanwhile, the hind part recorded the highest value when rabbits fed diet containing $4 \%$ CSS by $(47.89 \%)$ followed by that fed $6 \%$ CSS $(26.82 \%)$ and the that rabbits received $2 \%$ CSS containing diet by $(21.51 \%)$ compared to control diet $\left(\mathrm{R}_{1}\right) 370.33 \mathrm{gm}$. Omer et al. (2013b) noted that dietary treatment had no significant effect on carcass cuts except for hind leg that was significantly $(\mathrm{P}<0.05)$ affected by inclusion flaxseed in rabbit diets. Abd-El-Hady (2014) observed that the rabbits fed the experimental diets 300 and $400 \mathrm{gm}$ digestarom had significantly higher weight of Pre-slaughter, hot carcass and dressing percentages than control group by (5.2 and 6.3\%), (5.1 and 5.9\%) and (4.4 and 5.5\%), respectively. Ahmad and AbdelTawwab ( 2011) found that the caraway seed meal (CSM) supplementation enhanced fish growth over the control diet; the highest fish growth and feed utilization were obtained when fish fed on a diet containing $10 \mathrm{~g} \mathrm{CSM} / \mathrm{kg}$ diet.

Chemical analysis of carcass boneless was significantly $(\mathrm{P}<0.05)$ affected by incorporated CSS in rabbit diets. The crude protein was significant increase in diets containing CSS compared to control, the best result observed with diet 3 was77.53, followed diet 4 was $76.15 \%$, and the lowest value with diet 2 was 74.15 compared with control was $72.84 \%$, on the other hand, the ether extract and ash were significant decrease with diets containing CSS compared with control, the significantly lowest values EE and ash recorded with diet R3 were 20.28 and 2.19\%, compared with control 25.04 and $2.48 \%$, respectively.

Table (7): Carcass cuts and chemical analysis of carcass boneless of the experimental rabbits groups.

\begin{tabular}{|c|c|c|c|c|c|}
\hline \multirow[t]{2}{*}{ Item } & & \multicolumn{4}{|c|}{ Experimental diets } \\
\hline & & $\begin{array}{c}\mathrm{R}_{1} \text { (Control, } \\
0 \% \text { CSS) }\end{array}$ & $\begin{array}{c}\mathrm{R}_{2} \\
(2 \% \mathrm{CSS})\end{array}$ & $\begin{array}{c}\mathrm{R}_{3} \\
(4 \% \mathrm{CSS})\end{array}$ & $\begin{array}{c}\mathrm{R}_{4} \\
(6 \% \mathrm{CSS})\end{array}$ \\
\hline \multicolumn{6}{|c|}{ Carcass cuts (half carcass): } \\
\hline \multirow[t]{2}{*}{ Fore part } & $\begin{array}{l}\text { weight, } \mathrm{g} \\
\% \text { of } \mathrm{CW}_{1}\end{array}$ & $\begin{array}{c}343.33^{\mathrm{b}} \pm 17.38 \\
19.23^{\mathrm{b}} \pm 0.63\end{array}$ & $\begin{array}{c}470.67^{\mathrm{a}} \pm 51.17 \\
21.46^{\mathrm{ab}} \pm 1.11\end{array}$ & $\begin{array}{c}539.67^{\mathrm{a}} \pm 7.88 \\
22.15^{\mathrm{a}} \pm 0.87\end{array}$ & $\begin{array}{c}470.67^{\mathrm{a}} \pm 14.66 \\
20.71^{\mathrm{ab}} \pm 0.11\end{array}$ \\
\hline & weight, $g$ & $191.67^{\mathrm{b}} \pm 9.53$ & $241.67^{\mathrm{ab}} \pm 22.30$ & $273^{\mathrm{a}} \pm 20.42$ & $253.67^{\mathrm{ab}} \pm 24.46$ \\
\hline Middle part & $\%$ of $\mathrm{CW}_{1}$ & $10.72 \pm 0.35$ & $11.07 \pm 0.69$ & $11.13 \pm .26$ & $11.13 \pm 0.87$ \\
\hline \multirow[t]{2}{*}{ Hind part } & weight, $g$ & $370.33 c \pm 8.67$ & $450 \mathrm{~b} \pm 15.50$ & $547.67 \mathrm{a} \pm 34.97$ & $469.67 b \pm 11.10$ \\
\hline & $\%$ of $\mathrm{CW}_{1}$ & $20.74 \pm 0.31$ & $20.72 \pm 0.85$ & $22.39 \pm 0.75$ & $20.68 \pm 0.44$ \\
\hline \multicolumn{6}{|c|}{ Chemical analysis of carcass boneless (half carcass): } \\
\hline Moisture & & $75.12 \pm 1.4$ & $74.88 \pm 1.2$ & $74.80 \pm 0.2$ & $74.95 \pm 0.8$ \\
\hline \multicolumn{6}{|c|}{ Chemical composition on DM basis } \\
\hline Ether extract & & $25.04^{\mathrm{a}} \pm 8.0$ & $23.49^{\mathrm{b}} \pm 2.7$ & $20.28^{\mathrm{c}} \pm 3.9$ & $21.54^{\mathrm{bc}} \pm 30.3$ \\
\hline Ash & & $2.48^{\mathrm{a}} \pm 0.4$ & $2.36 a b \pm 0.8$ & $2.19 \mathrm{a} \pm 0.5$ & $2.31^{\mathrm{ab}} \pm 2.6$ \\
\hline Crude proteir & & $72.48^{\mathrm{c}} \pm 1.74$ & $74.15^{\mathrm{b}} \pm 4.49$ & $77.53^{\mathrm{a}} \pm 3.71$ & $76.15^{\mathrm{a}} \pm 5.11$ \\
\hline
\end{tabular}

\section{Physical composition of the best $9^{\text {th }}, 10^{\text {th }}$ and $11^{\text {th }}$ ribs of the experimental groups:}

Data of Table (8) showed that incorporation CSS in rabbit diets significantly $(\mathrm{P}<0.05)$ increased both of weight lean and lean \% of ribs weight (RW). On the other hand both weight and \% of RW of bone and fat was significantly $(\mathrm{P}<0.05)$ decreased. These results disagree with those Omar et al. (2013b) who observed that the dietary treatment had no significant effect $(\mathrm{P}>0.05)$ on physical composition of the best $9^{\text {th }}, 10^{\text {th }}$ and $11^{\text {th }}$ ribs, meanwhile, they noted that bone weight was affected by inclusion flaxseed at different levels in rabbit diets.

\section{Chemical analysis of best $9^{\text {th }}, 10^{\text {th }}$ and $11^{\text {th }}$ ribs of the experimental groups:}

Data of Table (9) showed that dietary treatment had significant effect $(\mathrm{P}>0.05)$ on chemical compositions of the best results $9^{\text {th }}, 10^{\text {th }}$ and $11^{\text {th }}$ ribs. Ether extract and ash contents were decreased, meanwhile, crude protein content was significantly $(\mathrm{P}<0.05)$ increased with incorporation CSS in the diets comparing to control that not contained CSS. 
Table (8): Physical composition of the $9^{\text {th }}, 10^{\text {th }}$ and $11^{\text {th }}$ ribs of the experimental rabbits groups.

\begin{tabular}{|c|c|c|c|c|c|}
\hline \multirow[t]{2}{*}{ Item } & & \multicolumn{4}{|c|}{ Experimental diets } \\
\hline & & $\begin{array}{c}\text { R) (Control, } \\
0 \% \text { CSS) }\end{array}$ & $\begin{array}{c}\mathrm{R}_{2} \\
(2 \% \mathrm{CSS})\end{array}$ & $\begin{array}{c}\mathrm{R}_{3} \\
(4 \% \mathrm{CSS})\end{array}$ & $\begin{array}{c}\mathrm{R}_{4} \\
(6 \% \mathrm{CSS})\end{array}$ \\
\hline \multicolumn{2}{|c|}{ Ribs weight, g (RW) } & $72^{\mathrm{c}} \pm 2.75$ & $85^{\mathrm{b}} \pm 2.57$ & $100^{\mathrm{a}} \pm 2.36$ & $88^{\mathrm{b}} \pm 2.20$ \\
\hline \multirow{2}{*}{ Lean } & weight, $\mathrm{g}$ & $58.37^{\mathrm{c}} \pm 1.13$ & $71.75^{\mathrm{b}} \pm 1.75$ & $87.00^{\mathrm{a}} \pm 0.98$ & $74.74^{\mathrm{b}} \pm 1.53$ \\
\hline & $\%$ of RW & $81.07^{c} \pm 3.71$ & $84.41^{\mathrm{b}} \pm 3.55$ & $87.00^{\mathrm{a}} \pm 3.42$ & $84.93^{\mathrm{b}} \pm 3.48$ \\
\hline \multirow{3}{*}{ Bone } & weight, $\mathrm{g}$ & $8.73^{\mathrm{a}} \pm 0.33$ & $8.48^{\mathrm{b}} \pm 0.30$ & $8.37^{\mathrm{c}} \pm 0.22$ & $8.57^{\mathrm{b}} \pm 0.29$ \\
\hline & $\%$ of RW & $12.13^{\mathrm{a}} \pm 0.92$ & $9.98^{\mathrm{b}} \pm 0.87$ & $8.37^{\mathrm{c}} \pm 0.77$ & $9.74^{\mathrm{b}} \pm 0.84$ \\
\hline & weight, $g$ & $4.90^{\mathrm{a}} \pm 0.23$ & $4.77^{\mathrm{ab}} \pm 0.28$ & $4.63^{\mathrm{b}} \pm 0.21$ & $4.69^{\mathrm{b}} \pm 0.22$ \\
\hline Fat & $\%$ of RW & $6.80^{\mathrm{a}} \pm 0.42$ & $5.61^{\mathrm{ab}} \pm 0.39$ & $4.63^{\mathrm{b}} \pm 0.34$ & $5.33^{\mathrm{b}} \pm 0.36$ \\
\hline
\end{tabular}

$a, b$ and $c:$ Means in the same row having different superscripts differ significantly $(P<0.05) . R_{l}$ : Control diet. $R_{2}$ : contained $2 \%$ from caraway seeds sieving. $R_{3}$ : contained $4 \%$ from caraway seeds sieving. $R_{4}$ : contained $6 \%$ from caraway seeds sieving.

Table (9): Chemical analysis of best $9^{\text {th }}, 10^{\text {th }}$ and $11^{\text {th }}$ ribs of the experimental rabbits groups.

\begin{tabular}{lcccc}
\hline Item & \multicolumn{4}{c}{ Experimental diets $(\%)$} \\
\cline { 2 - 5 } & $\mathrm{R}_{1}(\mathrm{Control}$, & $\mathrm{R}_{2}$ & $\mathrm{R}_{3}$ & $\mathrm{R}_{4}$ \\
& $0 \% \mathrm{CSS})$ & $(2 \% \mathrm{CSS})$ & $(4 \% \mathrm{CSS})$ & $(6 \% \mathrm{CSS})$ \\
\hline Moisture & $76.16 \pm 1.1$ & $75.71 \pm 0.8$ & $75.45 \pm 0.7$ & $75.34 \pm 0.3$ \\
Chemical analysis on DM basis & & & \\
Ether Extract (EE) & $26.33^{\mathrm{a}} \pm 1.82$ & $24.26^{\mathrm{a}} \pm 1.47$ & $22.56^{\mathrm{b}} \pm 1.22$ & $25.17^{\mathrm{a}} \pm 1.72$ \\
Ash & $3.16^{\mathrm{a}} \pm 0.2$ & $3.02^{\mathrm{a}} \pm 0.3$ & $2.72^{\mathrm{c}} \pm 0.4$ & $2.87^{\mathrm{b}} \pm 0.6$ \\
Crude protein (CP) & $70.51^{\mathrm{c}} \pm 1.91$ & $72.72^{\mathrm{b}} \pm 6.90$ & $74.72^{\mathrm{a}} \pm 1.45$ & $71.96^{\mathrm{b}} \pm 6.16$ \\
\hline a, b and c: Means in the same row having different superscripts differ significantly $(P<0.05)$. & $R_{1}:$ Control diet. $R_{2}:$ \\
contained $2 \%$ from caraway seeds sieving. $R_{3}:$ contained $4 \%$ from caraway seeds sieving. $R_{4}:$ contained $6 \%$ from \\
caraway seeds sieving.
\end{tabular}

The best result of chemical analysis was recorded by rabbits fed $4 \%$ CSS containing diet. These results disagree with those noted by Omer et al. (2013b) who observed that, dietary treatment had not significantly affect $(\mathrm{P}>0.05)$ on chemical analysis of the 9,10 and $11^{\text {th }}$ ribs when rabbits received diets contained different levels of flaxseed. Also, they reported that the dietary treatment had no significant effect $(\mathrm{P}>0.05)$ on chemical compositions (except ash $\%$ content) of the $9^{\text {th }}, 10^{\text {th }}$ and $11^{\text {th }}$ ribs.

\section{CONCLUSION}

From the results obtained it can be mentioned that incorporation caraway seeds sieving (CSS) in rabbit diets up to $4 \%$ could improve their dressing percentages and carcass characteristics without occurred any adverse effect on the other carcass parameters.

\section{REFERENCES}

Abd-El-Hady, A.M. (2014). Performance, physiological parameters and slaughter characteristics in growing rabbits as affected by herbal feed additives (digestarom ${ }^{\circledR}$ ). Journal of International Scientific Publications: Agriculture and Food Volume 2, ISSN 1314-8591 (Online), Published at: http://www.scientific- publications.net.

Abdo, Z.M.A.; A.Z.M. Soliman and O.S. Barakat (2003). Effect of hot pepper and marjoram as feed additives on the growth performance and the microbial population of the gastrointestinal tract of broilers. Egypt. Poult. Sci., 23: 91-113.

AOAC (2005). Official Methods of Analysis, $18^{\text {th }}$ ed. Association of Official Analytical Chemists, 
Washington, DC, USA.

Ahmad M. H., M. Abdel-Tawwab (2011). The use of caraway seed meal as a feed additive in fish diets: Growth performance, feed utilization, and whole-body composition of Nile tilapia, Oreochromis niloticus (L.) fingerlings. Aquaculture 110-114.

Blasco, A.; J. Quha yaun and G. Masoscro (1993). Hormonization of criteria and terminology in rabbit meat research. World Rabbits Sciences, 1: 3-10.

Blaxter, K.L. (1968). The energy metabolism of ruminants. $2^{\text {nd }}$ ed. Charles Thomas Publisher. Spring field. Illinois, U.S.A.

Çabuk, M.; M. Bozkurt; A. Alçiçek; Y. Akbaş and K. Küçükyılmaz (2006). Effect of a herbal essential oil mixture on growth and internal organ weight of broilers from young and old breeder flocks. South African Journal of Animal Science, 36 (2): 135-141.

Calsamiglia S., M.D. Stem and J.L. Frinkins (1995). Effects of protein source on nitrogen metabolism in continuous culture and intestinal digestion in vitro. Journal of Animal Science, 73:1819.

Chang, J. (2000). Medicinal herbs: drugs or dietary supplements? Biochemical Pharmacology $59,211-219$.

Deepak, S. (2013). Importance of Cuminum cyminum L. and Carum carvi L. in traditional medicaments. A review. Indian Journal of Traditional Knowledge, 12, 300-307.

Duncan, D.B. (1955). Multiple Rang and Multiple F-Test Biometrics,11:1- 42.

El-Dakar, A.Y. (2004). Growth response of hybrid tilapia, Oreochromis niloticus $x$ Oreochromis auraus to diets supplemented to different levels of caraway seeds. Agric. Sci. Mansoura Univ., 29 (11): 6083 6094.

El-Soud, A.; N. El-Lithy; G. El-Saeed; M. Wahby; M. Khalil; F. Morsy; N. Shaffie (2014). Reno protective effects of caraway (Carum carvi L.) essential oil in streptozotocin induced diabetic rats. Journal of Applied Pharmaceutical Science, 4, 27-33.

Fekete, S. and T. Gippert (1986). Digestibility and nutritive value of nineteen important feedstuffs for rabbits. J. Appli. Rabbit Res., 9 (3): 103- 108.

Ibrahim, Sh.A., A.A. El-Ghamry and G.M. El- Mallah (2000). Effect of some plants of Labiatae family as feed additives on growth and metabolic changes of rabbits. Egyptian J. Rabbit Sci., 10 (1): 105120.

Jafari, B. (2011). Influence of caraway on improved performance and blood parameters of Japanese quails. Annals of Biological Re-search, 2, 474-478.

NRC (1977). National Research Council. Nutrient requirements of rabbits. National Academy of Science, Washington, D.C.

Omer, H.A.A. Sawsan M. Ahmed, AbdEl-Maged A. Abedo and Azza M.M. Badr (2013b). Utilization of Flaxseeds (Linum usitatissimum L.) in Rabbit Rations. 2. Influence of Flaxseeds Levels supplementations on Blood Constituents, Carcass Characteristics and Fatty Acids Profile. Life Science Journal, 10 (4): 2625-2637.

Omer, H.A.A.; Hewida, M.H. Elallawy; Laila D. Abd El-Samee and Nagwa Maghraby (2010). Productive performance of rabbits fed diets containing lemongrass or active dried yeast. AmericanEurasian J. Agric. \& Environ. Sci., 7(2): 179-187.

Omer, H.A.A.; Y.A.A. EL-Nomeary; R.I. EL-Kady; Azza M.M. Badr; F.A.F. Ali, Sawsan M. Ahmed; H.M.H. EL-Allawy and Sh. A.M. Ibrahim (2013). Improving the Utilization of Rabbit Diets Containing Vegetable Oil by Using Fennel (Foeniculum vulgare) and Oregano (Origanum vulgare L) as Feed Additives. Life Science Journal. 10 (1): 2625-2636.

Peter, K. (2006). Handbook of herbs and spices (Wood head Publishing).

Platel, K., A. Rao, G. Saraswathi and K. Srinivasan (2002). Digestive stimulant action of three Indian

Radwan, N.L. and A.M. Abdel-Khalek (2007). Response of summer stressed growing rabbits to some dietary growth promoters. ISAH-Tartu, Estonia, 350-358.

Rahimi, S., Z. Teymouri Zadeh, M.A. Karimi Torshizi, R. Omidbaigi and H. Rokni (2011). Effect of the 
three herbal extracts on growth performance, immune system, blood factors and intestinal selected bacterial population in broiler chickens. J. Agr. Sci. Tech., 13: 527-539.

SAS (2002). Institute Inc., SYSTEM 2002® Software: Product Support Manual, Version 1, First Edition, Cary, NC: SAS Institute Inc.,

Sedlakova, J.; B. Kocourkova and V. Kuban (2001). Determination of the essential oil content and composition of caraway (Carum carvi L.). Czech J. Food Sci., 19: 31-36.

Valizadehm M. S; K. Kazemi Tabar and G.A. Nematzadeh (2007). A Novel for regeneration of plantlets from embryo explants of bunium persicum (Boiss.) B. Fedtsch. Int. J. Plant breeding and genet., 1(1):12-17.

Van Soest, P.J.; J.B. Robertson and B.A. Lewis (1991). Methods for dietary fiber, neutral detergent fiber and non- starch polysaccharides in relation to animal performance. Journal of Dairy Science, 74: 3583-3597, Cambridge,

Wichtl, M. (1994). Herbal Drugs and Phytopharmaceuticals. Boca Raton, CRC Press, FL, USA, pp. $128-129$.

Xiang, X. and Zhou, X.H. (2000). Application effect of Chinese herb medicine to aquatic animal feeds. Cereal and Feed Industry (China) 3, 27-29.

\section{تأثير غربلة بذور الكراوية (Carum carvi L.}

$$
\text { المركز الاقليمى لملاغنية والاعلاف- مركز البحوث الزراعية - جبيرة - مصر. }
$$

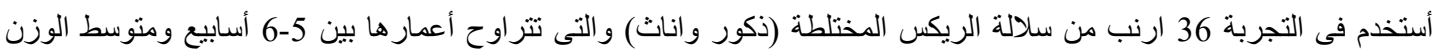

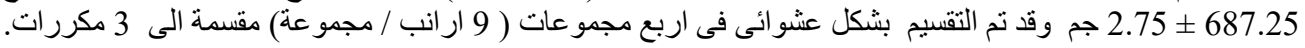

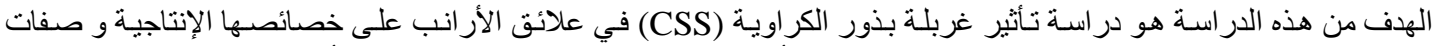

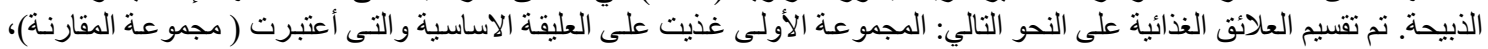

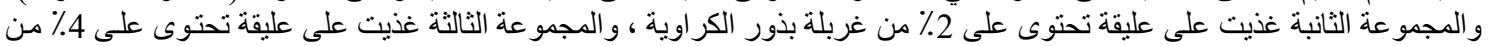

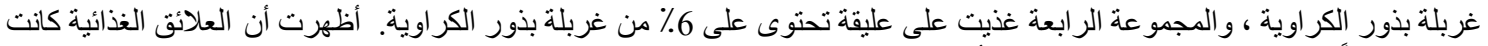

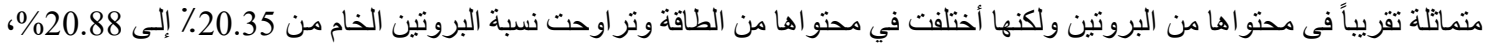

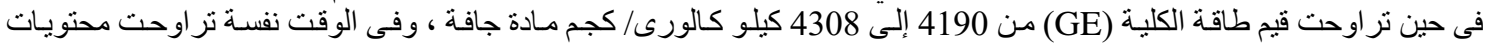

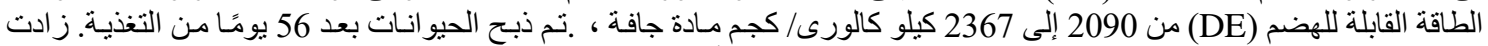

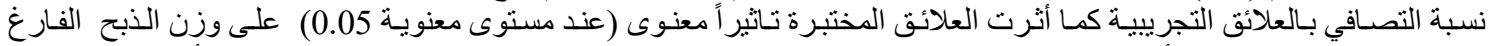

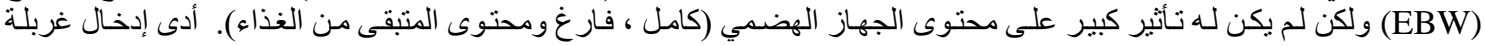

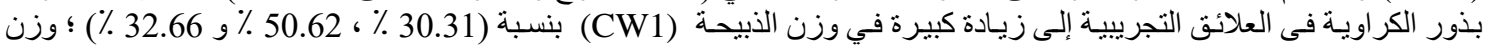

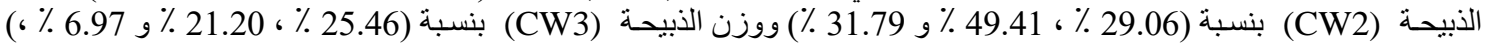

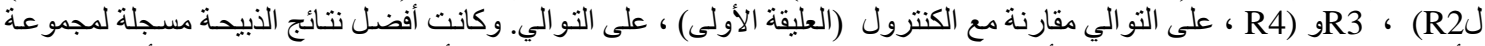

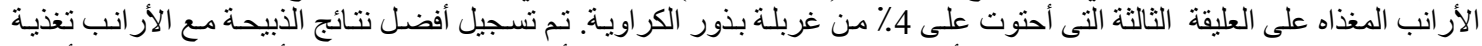

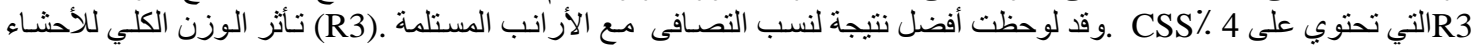

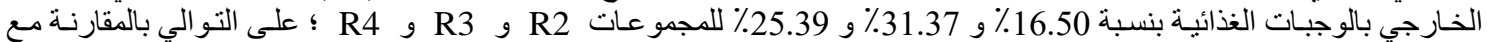

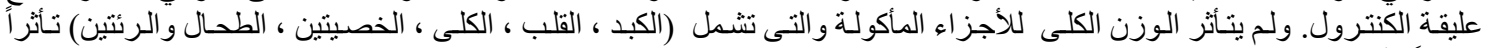

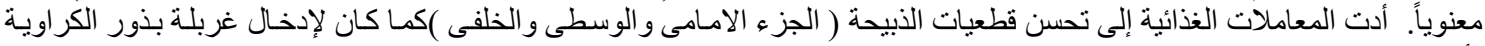

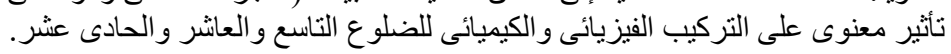

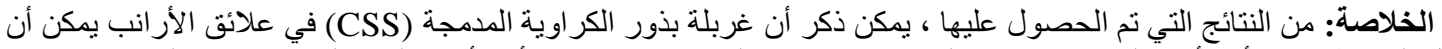
تصل إلى 4 ٪ حيث أنها أدت الى تحسن نسب التصافى وقياسات الذبيحة دون حدوث أي تأثير سلبي على خصائص الذيبـة الذيحة . 